\title{
Diabetic ketoacidosis in the dialysis-dependent patient: two case reports and recommendations for treatment
}

\author{
Jamie Blicker, MD; Anthony M. Herd, MD; Joanne Talbot, MD
}

\section{Introduction}

Diabetic ketoacidosis (DKA) is a complex medical disorder characterized by abnormalities in electrolyte, acid-base and volume status. DKA management is particularly challenging in patients with chronic renal failure on hemodialysis. Although the management of DKA is well-established, ${ }^{1,2}$ there is a paucity of literature describing DKA management in patients with concomitant renal failure requiring hemodialysis. A Medline search using a variety of search terms including "DKA AND renal failure," "DKA AND chronic renal failure," "DKA AND dialysis" and "DKA AND hemodialysis" returned only 2 relevant citations. ${ }^{3,4}$

This article describes 2 dialysis-dependent patients who presented to the emergency department (ED) with DKA, 1 patient with volume overload and 1 with dehydration. Presentation of these 2 case reports is followed by a discussion of key therapeutic issues.

\section{Case 1}

A 32-year-old woman with insulin dependent diabetes mellitus and chronic renal failure on thrice-weekly hemodialysis presented to our ED with nausea, vomiting and abdominal pain. She had taken no insulin for 24 hours because of anorexia. On presentation she had marked ketotic halitosis with Kussmaul's respirations. Her vital signs in- cluded a temperature of $36.0^{\circ} \mathrm{C}$, heart rate of 94 beats $/ \mathrm{min}$, respiratory rate of 20 breaths/min, blood pressure of 178/108 mm Hg, and oxygen saturation of 93\%. Clinical examination was unremarkable apart from bilateral lung crackles, an S4 gallop and distended jugular veins. Her hematocrit was $0.255 \mathrm{~g} / \mathrm{L}$, and total white blood cell count was $7.2 \times 10^{9} / \mathrm{L}$. Table 1 summarizes serum biochemistry and arterial blood gas results. Other relevant results included a $\beta$-hydroxy butyrate level of $9.1 \mathrm{mmol} / \mathrm{L}$, calcium of $2.73 \mathrm{mmol} / \mathrm{L}$, phosphate of $1.69 \mathrm{mmol} / \mathrm{L}$, and lipase of 1084 units/L.

The initial ECG showed wide QRS complexes, therefore she was treated speculatively for hyperkalemia. Her QRS complexes normalized after $1 \mathrm{~g}$ of calcium gluconate, 100 $\mathrm{mEq}$ of sodium bicarbonate and 8 units of regular insulin. She was started on a regular insulin infusion at 3 units $/ \mathrm{hr}$, which was titrated based on hourly bedside glucose checks. A chest x-ray showed marked fluid overload, therefore no additional crystalloid was administered.

Table 1 shows repeat biochemical results after 3 hours of ED therapy but before hemodialysis. Shortly thereafter, she was transferred to the intensive care unit (ICU), where she underwent hemodialysis and further insulin therapy. Her post-dialysis biochemical results are also illustrated in Table 1. Subsequently, an ultrasound ordered to investigate her abdominal pain revealed cholelithiasis, and she underwent laparoscopic cholecystectomy followed by an uneventful recovery. 


\section{Case 2}

A 47-year-old alcoholic man with insulin-dependent diabetes, hypertension and chronic renal failure on thriceweekly hemodialysis was brought to the ED by an acquaintance who found him bedridden at home. He had been drinking heavily for several days prior to presentation, but not eating nor taking his insulin or other medications. On examination, he was obtunded, he smelled strongly of ketones, and he had Kussmaul's respirations. His vital signs included a temperature of $36.3^{\circ} \mathrm{C}$, pulse of 96 beats/min, respiratory rate of 20 breaths/min, blood pressure of 225/145 mm Hg, and oxygen saturation of $99 \%$. He appeared dehydrated, with flat external jugular veins, dry mucous membranes and a clear chest on auscultation. The remainder of his clinical examination revealed a coarse systolic ejection murmur and abnormal bilateral plantar responses, but no other significant findings.

His initial biochemical and arterial blood gas results are illustrated in Table 1. Additionally, his serum $\beta$-hydroxy butyrate was $6.2 \mathrm{mmol} / \mathrm{L}$, serum calcium was 1.60 $\mathrm{mmol} / \mathrm{L}$, serum phosphate was $1.99 \mathrm{mmol} / \mathrm{L}$, serum lactate was $4.5 \mathrm{mmol} / \mathrm{L}$, hematocrit was $0.236 \mathrm{~g} / \mathrm{L}$, and total white blood cell count was $21.0 \times 10^{9} / \mathrm{L}$. An ECG showed an old right bundle branch block, and his chest x-ray was unremarkable, with no signs of volume overload.

In the ED, he received a bolus of 10 units of regular insulin followed by an infusion at 5 units/hr, which was titrated based on hourly bedside tests. Additionally, he received four $500-\mathrm{mL}$ aliquots of normal saline. His level of consciousness and overall clinical condition improved. Repeat biochemical and arterial blood gas results after 2 hours of treatment in the ED are shown in Table 1. He was admitted to the ICU and dialyzed shortly thereafter. Postdialysis biochemical results are also shown in Table 1.

\section{Discussion}

\section{DKA and chronic renal failure}

Long-standing or poorly controlled diabetes often leads to chronic renal failure requiring hemodialysis. Renal disease is a leading cause of death and disability in diabetic patients, and disease management becomes increasingly complex in anuric patients. ${ }^{5}$ DKA and complications of chronic renal failure are common reasons for patients to present to acute care settings, but despite substantial overlap between diabetes and chronic renal failure, the occurrence of DKA in hemodialysis-dependent patients is quite rare, even in high volume tertiary care referral and dialysis centres such as ours. Research and epidemiological data in this area are conspicuously absent and a recent literature search, using Medline and HealthSTAR databases, revealed only 1 review article and 1 case report. ${ }^{3,4}$

\section{Metabolic considerations}

Chronic renal failure affects virtually every organ system; therefore, dialysis patients can present to the ED with a multitude of problems. ${ }^{6-9}$ Among the most common are fluid overload and hyperkalemia due to difficulty adhering to the strict dietary restrictions that must be followed, especially on non-dialysis days. Acidosis frequently occurs because of the kidneys' inability to excrete the daily metabolic acid load, and hyperphosphatemia and hypocalcemia result from phosphate retention and inade-

Table 1. Biochemical test results* for 2 dialysis-dependent patients

\begin{tabular}{|c|c|c|c|c|c|c|}
\hline \multirow[b]{2}{*}{ Type of test } & \multicolumn{3}{|c|}{ Case 1} & \multicolumn{3}{|c|}{ Case 2} \\
\hline & $\begin{array}{c}\text { On } \\
\text { arrival }\end{array}$ & $\begin{array}{c}\text { Before } \\
\text { hemodialysis }\end{array}$ & $\begin{array}{c}\text { After } \\
\text { hemodialysis }\end{array}$ & $\begin{array}{c}\text { On } \\
\text { arrival }\end{array}$ & $\begin{array}{c}\text { Before } \\
\text { hemodialysis }\end{array}$ & $\begin{array}{c}\text { After } \\
\text { hemodialysis }\end{array}$ \\
\hline Sodium & 110 & 122 & 138 & 120 & 122 & 137 \\
\hline Potassium & 9.8 & 6.3 & 3.7 & 5.8 & 4.2 & 3.3 \\
\hline Chloride & 74 & 81 & 101 & 80 & 84 & 99 \\
\hline $\mathrm{CO}_{2}$ & 4 & 16 & 29 & 9 & 13 & 27 \\
\hline Glucose & 78.2 & 66.2 & 23.5 & 37.7 & 24.8 & 26.9 \\
\hline Urea & 22.4 & 23.1 & 8.1 & 34.3 & 34.7 & 20.0 \\
\hline Creatinine & 672 & 686 & 272 & 783 & 775 & 492 \\
\hline $\mathrm{PaO}_{2}$ & 123 & - & - & 118 & 122 & - \\
\hline $\mathrm{PaCO}_{2}$ & 19 & - & - & 23 & 26 & - \\
\hline $\mathrm{pH}$ & 7.2 & - & - & 7.13 & 7.2 & - \\
\hline $\mathrm{HCO}_{3}$ & 7.1 & - & - & 8 & 10 & - \\
\hline
\end{tabular}

*Results in $\mathrm{mmol} / \mathrm{L}$ or $\mathrm{mm} \mathrm{HG}$, except $\mathrm{pH}$. 
quate renal vitamin D synthesis. In addition to these metabolic and electrolyte disturbances, patients often suffer from anorexia, nausea, vomiting, infections, and even acute coronary events. ${ }^{10,11}$

\section{DKA and hemodialysis}

There are several reasons why DKA is uncommon in hemodialysis patients. Although most patients with chronic renal failure have impaired glucose tolerance, the kidneys play a major role in insulin breakdown, therefore many patients see an improvement in glycemic control when they progress to hemodialysis. ${ }^{12}$ As well, because glycosuria and osmotic diuresis account for most of the fluid and electrolyte losses seen in DKA, anuric patients may be somewhat protected from dehydration and shock, although still subject to hyperkalemia and metabolic acidosis. This said, substantial volume loss, as described in Case 2, can still occur due to a prolonged decrease in oral intake or increased insensible water losses related to tachypnea and fever.

Another plausible explanation for the relative rarity of DKA in hemodialysis patients is that these patients are typically seen 2 to 3 times weekly for dialysis, so diabetic decompensation may be recognized and treated quickly. Moreover, patients who are able to comply with the lifestyle and dietary demands of hemodialysis are perhaps a more informed and motivated group, better able to manage their diabetes effectively.

\section{Treatment}

DKA management is well described elsewhere, but existing treatment guidelines do not address management of the anuric patient, ${ }^{1,2}$ several key principles differ in this setting, and there are no evidence-based guidelines. The following recommendations are based upon current understanding of DKA and chronic renal failure pathophysiology.

\section{Recommendation \#1}

Dialysis dependent patients with DKA should be presumed to be hyperkalemic and should not receive routine potassium supplementation. Even when testing reveals hypokalemia, total body potassium stores may be high, and these patients are unable to excrete a potassium load. Consequently, hypokalemia must be documented and acidosis corrected before potassium supplementation is initiated. All dialysis patients presenting with significant symptoms should undergo immediate cardiac monitoring. If there is clinical suspicion or electrocardiographic evidence of hyperkalemia, they should receive immediate potassium lowering therapies, including emergent hemodialysis. ${ }^{8}$

\section{Recommendation \#2}

Dialysis dependent patients with DKA may not be volume depleted. If volume resuscitation is required, it should involve frequent small aliquots with continuous re-evaluation. Consideration should be given to invasive monitoring. When volume overload is apparent, immediate hemodialysis is the therapy of choice.

\section{Recommendation \#3}

Anuric kidneys cannot manage normal daily acid production, and DKA is associated with ketogenesis and increased acid loading; therefore, dialysis-dependent patients with DKA often present with profound metabolic acidosis. In addition, pulmonary dysfunction related to volume overload and underlying lung conditions such as pneumonia can impair ventilatory compensation to metabolic acidosis. In DKA victims with functioning kidneys, aggressive fluid resuscitation can partially correct metabolic acidosis by increasing renal blood flow and hence acid excretion, but this is not an option in dialysis-dependent patients. Bicarbonate administration is rarely of value in DKA, ${ }^{13}$ and the associated volume, sodium and osmotic overload may be particularly problematic for anuric patients. In this situation, significant metabolic acidosis will only be correctable by hemodialysis. ${ }^{4}$

\section{Recommendation \#4}

Dialysis patients are subject to numerous conditions that precipitate DKA; therefore, in hemodialysis-dependent patients it is important to search for a precipitating cause. In particular, their compromised immune status, constant state of protein malnutrition and frequent vascular accessing predispose them to significant infections. ${ }^{10}$ Cardiovascular disease is also extremely common, ${ }^{11}$ and evidence of myocardial ischemia should be actively sought and excluded. Many such patients will require hospital admission for further investigation and management of DKA.

\section{Conclusions}

DKA is relatively uncommon in hemodialysis-dependent patients, and DKA management must be fundamentally altered in these patients, who are likely to have different fluid and electrolyte needs than other patients with DKA. In anuric patients with DKA, significant hyperkalemia, volume overload and acidosis suggest the need for urgent or emergent hemodialysis.

Competing interests: None declared.

Key words: diabetes; ketoacidosis; renal failure; dialysis 


\section{References}

1. Magee MF, Bhatt BA. Management of decompensated diabetes. Diabetic ketoacidosis and hyperglycemic hyperosmolar syndrome. Crit Care Clin 2001;17(1):75-106.

2. Delaney MF, Zisman A, Kettyle WM. Diabetic ketoacidosis and hyperglycemic hyperosmolar nonketotic syndrome. Endocrinol Metab Clin North Am 2000;29(4):683-705.

3. Catalano C, Fabbian F, Di Landro D. Acute pulmonary oedema occurring in association with diabetic ketoacidosis in a diabetic patient with chronic renal failure. Nephrol Dial Transplant 1998;13(2):491-2.

4. Tzamaloukas AH, Avasthi PS. Acid-base disorders in hyperglycemia of insulin-dependent diabetic patients on chronic dialysis. J Diabet Complications 1988;2(2):75-8.

5. Port FK. Morbidity and mortality in dialysis patients. Kidney Int 1994;46(6):1728-37.

6. Eadington D, Plant W, Winney R. Chronic renal failure. Practitioner 1993;237(1522):64-9.

7. Jacobson HR. Chronic renal failure: pathophysiology. Lancet 1991;338:419-23.
8. Klahr S. Chronic renal failure: management. Lancet 1991; 338:423-7.

9. Loran MJ, McErlean M, Eisele G, Raccio-Robak N, Verdile VP. The emergency department care of hemodialysis patients. Clin Nephrol 2002;57(6):439-43.

10. Minnaganti VR, Cunha BA. Infections associated with uremia and dialysis. Infect Dis North Am 2001;15(2):385-406.

11. Alpert MA. Cardiovascular factors influencing survival in dialysis patients. Adv Perit Dial 1996;12:110-9.

12. Mak RH. Impact of end-stage renal disease and dialysis on glycemic control. Semin Dial 2000;13(1):4-8.

13. Viallon A, Zeni F, Lafond P, Tardy B, Page Y, Bertrand JC. Does bicarbonate therapy improve the management of severe diabetic ketoacidosis? Criti Care Med 1999;27:2690-3.

Correspondence to: Dr. Anthony M. Herd, GF-201, Health Sciences Centre, 820 Sherbrook St., Winnipeg MB R3A 1R9; 204 787-2934, fax 204 787- 5134, am.herd@shaw.ca 